\title{
Predicting hypoxaemia during flights in children with cystic fibrosis
}

\author{
R M Buchdahl, A Babiker, A Bush, D Cramer
}

\begin{abstract}
Background-We have previously suggested that it is possible to predict oxygen desaturation during flight in children with cystic fibrosis and chronic lung disease by non-invasive measurement of oxygen saturation following inhalation of $15 \%$ oxygen-the pre-flight hypoxic challenge. This study reports on the results of measurements over 5 years.

Methods-The study comprised a preflight hypoxic challenge measuring oxygen saturation by finger tip pulse oximetry $\left(\mathrm{Spo}_{2}\right)$ during tidal breathing of $15 \%$ oxygen in nitrogen and spirometric testing 1 month before the flight followed by $\mathrm{Spo}_{2}$ measurements during intercontinental flights to and from holidays abroad with children in wake and sleep states.
\end{abstract}

Results-Pre-flight tests were completed on 87 children with cystic fibrosis. Desaturation of $<90 \%$ occurred in 10 children at some stage during the flight, three of whom received supplementary oxygen. Using a cut off $\mathrm{SpO}_{2}$ of $90 \%$, the pre-flight hypoxic challenge correctly predicted desaturation in only two of these children. The sensitivity and specificity of the pre-flight hypoxic challenge were $20 \%$ and $99 \%$, respectively, compared with $70 \%$ and $\mathbf{9 6 \%}$ for spirometric tests (using a cut off for forced expiratory volume in 1 second $\left(\mathrm{FEV}_{1}\right)$ of $<50 \%$ predicted). Overall, preflight spirometric tests were a better predictor of desaturation during flight with the area under the Receiver Operating Characteristic (ROC) curve of 0.89 compared with 0.73 for the hypoxic challenge test.

Conclusions-In this group of subjects pre-flight spirometric testing was a better predictor of desaturation during flight than the pre-flight hypoxic challenge.

(Thorax 2001;56:877-879)

Keywords: cystic fibrosis; flying; travel; desaturation; children

Passenger cabins of commercial aircraft are pressurised during flight to an altitude of 1525-2440 metres (5000-8000 feet), equivalent to breathing $17.2-15.1 \%$ oxygen at sea level. We have previously suggested that it is possible to predict oxygen desaturation during a flight in children with cystic fibrosis by noninvasive measurement of oxygen saturation $\left(\mathrm{SpO}_{2}\right)$ following inhalation of $15 \%$ oxygen, the pre-flight hypoxic challenge. ${ }^{1}$ The test appeared to be better at predicting desaturation during flight than spirometric tests. This study evaluates the outcome of the test over a 5 year period.

\section{Methods}

Children with cystic fibrosis with a history of requiring supplementary oxygen or who were sputum positive for Burkholderia cepacia and/or methicillin resistant Staphylococcus aureus were not accepted on holidays. One month before the flight children were seen at Hillingdon Hospital, Middlesex (approximately 12 metres above sea level) where they underwent spirometric tests using a Compact Vitalograph. Measurements included forced expiratory volume in 1 second $\left(\mathrm{FEV}_{1}\right)$ expressed as percentage predicted (\%PR) values for sex, height, and age. ${ }^{2}$ Children then underwent the pre-flight hypoxic challenge by breathing a mixture of $15 \%$ oxygen and $85 \%$ nitrogen. Details of the test have been described previously. ${ }^{1}$ Following the challenge any child whose $\mathrm{SpO}_{2}$ measured less than $86 \%$ was advised not to fly.

The same portable oximeter used for the pre-flight challenge was used to measure $\mathrm{SpO}_{2}$ (Nellcor N-20) during flight. A single measurement was made at cruising altitude for both outward and return journeys approximately half an hour into the flight. Where children fell asleep during the flight an additional $\mathrm{SpO}_{2}$ measurement was made while they were asleep. Any child who desaturated underwent further measurements during the flight.

\section{STATISTICAL ANALYSIS}

Sensitivity, specificity, positive and negative predictive values were calculated with exact binomial 95\% confidence intervals using an arbitrary cut off of $90 \%$ for the pre-flight hypoxic challenge and $\mathrm{FEV}_{1}<50 \% \mathrm{PR}$ for spirometric testing. Values were calculated on the basis of whether a child desaturated during any stage of the outward or return flight. The pre-flight hypoxic challenge and spirometric tests were compared by plotting Receiver Operating Characteristic (ROC) curves which measured sensitivity against (1 - specificity) - 
that is, the probability of a false positive-at different cut off values for each test. ${ }^{3}$ The curve starts at $(0,0)$ corresponding to a cut off with sensitivity 0 and specificity 1 , and continues to $(1,1)$ corresponding to a cut off with sensitivity 1 and specificity 0 . A test score with no predictive power would have an ROC curve coinciding with the line whose slope $=1$. The greater the predictive power, the more bowed the ROC curve - that is, the closer to the point $(0,1)$ and the larger the area under the curve. Calculation of the ROC curve was implemented using sTATA (Stata Corporation, Texas, 1999).

\section{Results}

Eighty seven children (40 boys) aged 7-19 years with cystic fibrosis diagnosed using conventional criteria participated in nine separate holidays (six to the USA, two to the Caribbean, and one to Mauritius). Flight durations varied between 8 hours (USA) and 13 hours (Mauritius). The airline carriers were American Airlines (MD11, Boeing 767 and 777), Virgin (MD11), and Air Mauritius (A340 airbus). One boy went on holiday twice. Five of the 87 children who underwent pre-flight testing did not subsequently fly; in two the $\mathrm{SpO}_{2}$ measured during the pre-flight hypoxic challenge was below $86 \%$, one did not fly for social reasons, in one Burkholderia cepacia was identified in the sputum, and the fifth child fell ill on the day of travel. Eighty two children were measured in the awake state during the outward flight (data on one of these was lost). Thirty eight were also measured in the sleep state. On the return flight there were 81 children (one child returned on a separate flight), 73 of whom were measured while awake and while asleep. Eight children fell asleep before the awake measurements could be made.

A total of 10 children desaturated $<90 \%$ at some point during the outward and return flights. The pre-flight hypoxic challenge correctly predicted desaturation in only two (test sensitivity $20 \%$ ( $95 \%$ CI 3 to $56 \%$ ), specificity $99 \%$ (95\% CI 93 to $100 \%$ ), positive predictive value $66 \%$ (95\% CI 9 to $99 \%$ ), and negative predictive value $90 \%$ (95\% CI 81 to $96 \%)$ ). In contrast, spirometric testing predicted desaturation in seven (sensitivity $70 \%$ (95\% CI 35 to

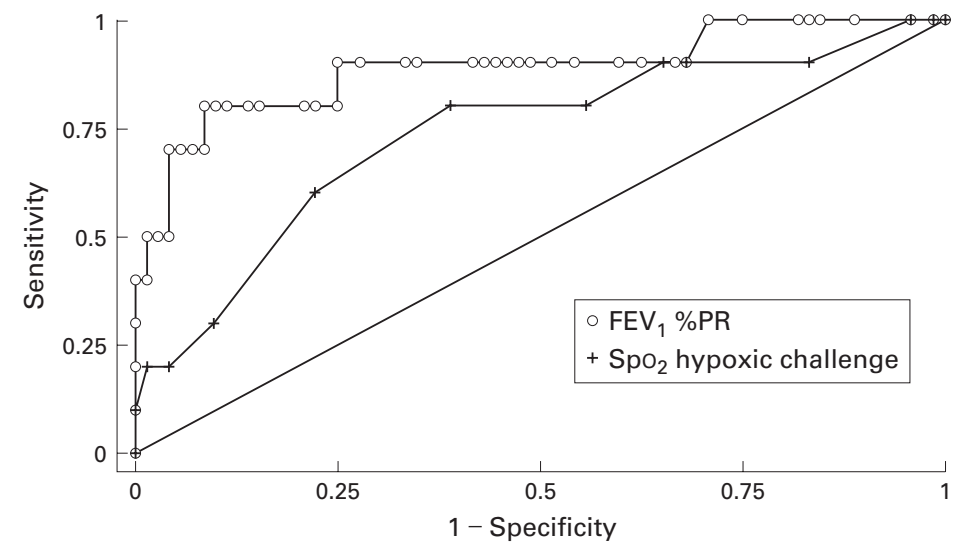

Figure 1 Receiver operating characteristic (ROC) curves comparing pre-flight hypoxic challenge $\left(\mathrm{SpO}_{2}\right)$ with spirometry $\left(\mathrm{FEV} \mathrm{1}_{1} \% \mathrm{PR}\right)$ by plotting the sensitivity against 1 specificity (the probability of a false positive) for each test.
95\%), specificity $93 \%$ (95\% CI 85 to $98 \%$ ), positive predictive value $58 \%$ ( $95 \%$ CI 28 to $85 \%)$, and negative predictive value $96 \%(95 \%$ CI 88 to $99 \%)$ ). Figure 1 shows the ROC curves for the pre-flight hypoxic challenge and spirometric tests using $\mathrm{FEV}_{1} \% \mathrm{PR}$. For $\mathrm{FEV}_{1} \% \mathrm{PR}$ the area under the curve was 0.89 compared with 0.73 for the pre-flight hypoxic challenge. When both pre-flight spirometric and hypoxic challenge tests were combined, using the same cut off values, inflight desaturation of $<90 \%$ was correctly predicted in one extra child - that is, a true positive rate of 8 out of 10). The false positive rate was 5 ( 5 for pre-flight spirometric testing and 1 for hypoxic challenge). Combining spirometric testing with hypoxic challenge therefore only marginally improved the discriminatory value of either test on its own.

Following the first holiday to America when one boy became hypoxic, the airline carriers were informed about children whose pre-flight $\mathrm{SpO}_{2}$ was $<90 \%$ and advised to carry extra oxygen to be administered at a rate of $21 / \mathrm{min}$ via nasal cannulae on board the flight for those individuals if they desaturated $<90 \%$. Of the 10 children who desaturated, five felt unwell with breathlessness. This settled following inhalation of supplementary oxygen. However, one child felt uncomfortable inhaling oxygen via nasal cannulae and refused to use it. Five of the children who desaturated felt well with no obvious signs and declined to use oxygen when offered. No child with an $\mathrm{Spo}_{2}$ of $90 \%$ or more during the flight was noted to be symptomatic or to require oxygen.

\section{Discussion}

The results of this study suggest that, for this selected group of subjects, neither the preflight hypoxic test nor pre-flight spirometric testing is a good predictor of desaturation during flight, although spirometric testing proved more discriminatory overall. The findings do not necessarily pertain for other chronic lung or cardiopulmonary disease groups, or for an adult population. It should also be noted that the prevalence of desaturation of $<90 \%$ during flight was low, occurring in only 10 children.

In contrast to our original study, ${ }^{1}$ we found that pre-flight spirometric testing was a better predictor of inflight desaturation than the preflight hypoxic challenge. There were, however, design differences between the two studies. There were only 22 children in the original study all over the age of 11 years. In that study $\mathrm{SpO}_{2}$ measurements were performed on mountain slopes as well as during flight and no child was measured in the sleep state when further falls in saturation might be expected. Other authors have found that spirometric tests accurately predict hypoxia during flight. ${ }^{4}$ Spirometry measures flow rates and dynamic volumes which may correlate better with the physiological variables that affect alveolar capillary oxygen transfer such as age, ventilatory drive, ventilation/perfusion mismatching, and ventilatory impedance. Hyperventilation in response to desaturation during the pre-flight hypoxic challenge might explain the high false negative rate of the test. 
In conclusion, we found that pre-flight spirometric testing in this group of young people with cystic fibrosis was a better predictor of in-flight hypoxia than the pre-flight hypoxic test. There was little to be gained by combining the two tests.

We are grateful to the members of The Cystic Fibrosis Holiday Fund for Children who helped organise the holidays and who also helped raise the necessary funds for the holidays and the pre-flight hypoxic challenge test equipment.
1 Oades PJ, Buchdahl RM, Bush A. Prediction of hypoxaemia at high altitude in children with cystic fibrosis. $B M \mathcal{F}$ 1994;308:15-8.

2 Polgar G, Weng TR. The functional development of the respiratory system from the period of gestation to adulthood. Am Rev Respir Dis 1979; 120:625-95.

3 Hanley JA, McNeil BJ. The meaning and use of the area under a receiver operating characteristic (ROC) curve. Radiology 1982;143:29-36.

Radiology 1982;143:29-36.
4 Dillard TA, Rosenberg AP, Berg BW. Hypoxemia during altitude exposure. A meta-analysis of chronic obstructive pulmonary disease. Chest 1993;103:422-5.

5 Miles JF, Ayres JG. Travel and respiratory disease: travel medicine. Travel Med Int 1990;8:147-50.

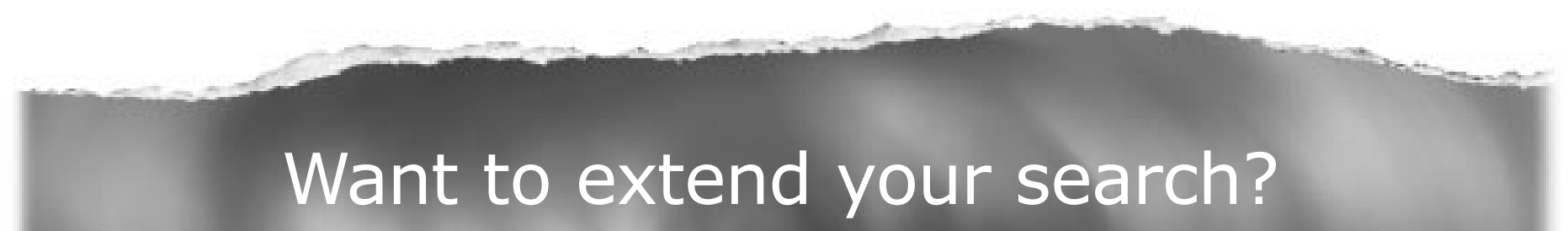

Cross journal searching

If you can't find what you are looking for in Thorax you can extend your search across many of the more than 200 journals available for selection. You can restrict your search to specific subject areas

(eg, clinical medicine, basic research), or select specific journals, or search all available titles.

\section{www.thoraxjnl.com}

